\title{
Is Needle Biopsy Clinically Useful in Preoperative Grading of Central Chondrosarcoma of the Pelvis and Long Bones?
}

\author{
Pablo D. Roitman MD, Germán L. Farfalli MD, Miguel A. Ayerza MD, \\ D. Luis Múscolo MD, Federico E. Milano MSC, Luis A. Aponte-Tinao MD
}

Published online: 16 February 2016

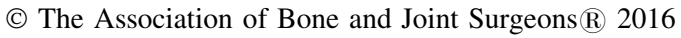

\begin{abstract}
Background Central chondrosarcoma of bone is graded on a scale of 1 to 3 according to histological criteria. Clinically, these tumors can be divided into low-grade (Grade 1) and high-grade (Grade 2, Grade 3, and dedifferentiated) chondrosarcomas. Although en bloc resection has been the most widely used treatment, it has become generally accepted that in selected patients with low-grade chondrosarcomas of long bones, curettage is safe and effective. This approach requires an accurate preoperative
\end{abstract}

One of the authors certifies that he (LAA-T) or a member of his immediate family, has or may receive payments or benefits, during the study period, an amount of USD 10,000 to USD 100,000 from Stryker Americas (Miramar, FL, USA).

All ICMJE Conflict of Interest Forms for authors and Clinical Orthopaedics and Related Research ${ }^{\mathbb{R}}$ editors and board members are on file with the publication and can be viewed on request.

Each author certifies that his institution has approved the reporting of this study and that all investigations were conducted in conformity with ethical principles of research.

This work was performed at the Italian Hospital of Buenos Aires, Buenos Aires, Argentina.

P. D. Roitman $(\bowtie)$

Pathology Department, Italian Hospital of Buenos Aires, Juan D. Perón 4190, 1199 Buenos Aires, Argentina

e-mail: pablo.roitman@hospitalitaliano.org.ar

G. L. Farfalli, M. A. Ayerza, D. L. Múscolo, L. A. Aponte-Tinao Institute of Orthopedics "Carlos E. Ottolenghi”, Italian Hospital of Buenos Aires, Buenos Aires, Argentina

F. E. Milano

Department of Bioengineering, Instituto Tecnologico de Buenos

Aires, Buenos Aires, Argentina

F. E. Milano

CONICET (Consejo Nacional de Investigaciones Cientificas y

Tecnicas), Buenos Aires, Argentina estimation of grade to avoid under- or overtreatment, but prior reports have indicated that both imaging and biopsy do not always give an accurate prediction of grade.

Questions/purposes (1) What is the concordance of image-guided needle preoperative biopsy and postoperative grading in central (intramedullary) chondrosarcomas of long bones, and how does this compare with the concordance of image-guided needle preoperative biopsy and postoperative grading in central pelvic chondrosarcomas? (2) What is the concordance of preoperative image-guided needle biopsy and postoperative findings in differentiating low-grade from high-grade central chondrosarcomas of long bones, and how does this compare with the concordance in central pelvic chondrosarcomas?

Methods Between 1997 and 2014, in our institution, we treated 126 patients for central chondrosarcomas located in long bones and the pelvis. Of these 126 cases, 41 were located in the pelvis and the remaining 85 cases were located in long bones. This study considers 39 (95\%) and $40(47 \%)$ of them, respectively. We included all cases in which histological information was complete regarding preoperative and postoperative tumor grading. We excluded all cases with incomplete data sets or nondiagnostic preoperative biopsies. To evaluate the needle biopsy accuracy, we compared the histological tumor grade, obtained from the preoperative biopsy, with the final histological grade obtained from the postoperative surgical specimen. The weighted and nonweighted kappa statistics were used to evaluate the agreement.

Results Concordance between the preoperative biopsy and the final pathological analysis in terms of histological grade was much higher in long-bone chondrosarcoma than in pelvic chondrosarcoma (83\% [33 of 40] versus 36\% [14 of 39]; odds ratio, 8, 48). Likewise, the weighted kappa coefficients were higher in long-bone chondrosarcoma than 
in pelvic chondrosarcoma for the determination of histological grade $(0.63$; $95 \%$ confidence interval [CI], 0.340.91 versus $0.12 ;-0.32$ to $0.57 ; \mathrm{p}<0.001)$. When categorizing the lesions as low grade or high grade, concordance between the preoperative biopsy and the final pathological analysis was much higher in long-bone chondrosarcoma than in pelvic chondrosarcoma (90\% [36 of 40] versus $67 \%$ [26 of 39]; odds ratio, 4, 5). Likewise, the weighted kappa coefficients were higher in long-bone chondrosarcoma than in pelvic chondrosarcoma $(0.73 ; 95 \%$ CI, 0.51-0.94 versus 0.26; 0.04-0.48; $\mathrm{p}<0.001$ ).

Conclusions Image-guided needle biopsy, when performed by a specialist radiologist and evaluated by an experienced bone pathologist, is a useful tool in determining the histological grade of long-bone chondrosarcomas allowing identification of true low-grade tumors. The histological grade should be correlated with imaging and the clinical presentation, but under these circumstances, experienced tumor surgeons may use this information in planning surgical treatment. The same appears not to be true for pelvic lesions, in which histological grade established by needle biopsy should be interpreted with caution.

Level of Evidence Level III, diagnostic study.

\section{Introduction}

Chondrosarcoma is a malignant neoplasm with hyaline cartilage differentiation [3]. It is graded on a scale of 1 to 3 according to nuclear size, nuclear staining, and cellularity $[3,10,24]$. These grading systems correlate well with their clinical behavior [1, 10, 17], which ranges from slowly growing, rarely metastasizing tumors (Grade 1, now termed atypical cartilaginous tumor/Grade 1 chondrosarcoma to stress that it behaves as a locally aggressive lesion and only metastasizes in exceptional cases) to very aggressive, metastasizing sarcomas (Grade 3). Dedifferentiated chondrosarcoma is defined by the presence of a biphasic tumor composed of a conventional chondrosarcoma (usually Grade 1) and a high-grade, noncartilage-producing sarcoma [3]. Clinically these tumors can be divided into low grade (Grade 1) and high grade (Grade 2, Grade 3, and dedifferentiated) [9].

There is considerable interobserver variability among pathologists when dealing with cartilaginous tumors $[8,22$, 23]; therefore, the role of preoperative biopsy has been controversial. Clinical manifestations and radiological features are used, alone or correlated with histology, to make therapeutic decisions $[2,5,7,11,14,22,27]$. Surgery remains the only curative treatment for patients with central (intramedullary) chondrosarcoma. En bloc resection is the most widely used treatment but, according to many recent studies, curettage is safe and effective in selected patients with low-grade chondrosarcoma of long bones [5, $12,16,18,26]$. Ideally, this approach would be based on accurate preoperative grading to avoid under- or overtreatment. In our clinical practice we have noticed some differences in preoperative versus postoperative grading between chondrosarcomas of long bones and pelvic chondrosarcomas with the pelvic group having less reliable results.

The aim of this study, therefore, was to determine the clinical utility of needle biopsy in preoperative grading of pelvic and long-bone chondrosarcomas. To evaluate the biopsy concordance, we attempted to answer the following questions: (1) What is the concordance of image-guided needle preoperative biopsy and postoperative grading in central chondrosarcomas of long bones, and how does this compare with the concordance of image-guided needle preoperative biopsy and postoperative grading in central pelvic chondrosarcomas? (2) What is the concordance of preoperative image-guided needle biopsy and postoperative findings in differentiating low-grade from high-grade central chondrosarcomas of long bones, and how does this compare with the concordance in central pelvic chondrosarcomas?

\section{Materials and Methods}

Patient information regarding preoperative and postoperative tumor grading was retrieved from the files of our institution. We included all cases in which histological information was complete regarding preoperative and postoperative tumor grading. We excluded all cases with incomplete data sets or nondiagnostic preoperative biopsies. Between 1997 and 2014, in our institution, we treated 126 patients for central chondrosarcomas located in long bones and the pelvis. Of these 126 cases, 41 were located in the pelvis and the remaining 85 cases were located in long bones. This study considers 39 pelvic cases $(95 \%)$ and 40 long-bone cases (47\%). Two pelvic chondrosarcomas (5\%) and 45 extremity tumors (53\%) were excluded. Of the 47 excluded cases, 21 (19 long bone and two pelvic cases) were excluded because of incomplete data sets. The remaining 26 long bones cases were excluded because of nondiagnostic preoperative biopsies. No patient included in this study was lost to followup.

The mean age at presentation was 47 years (range, 1779 years) with a female-to-male ratio of $1.5: 1$ for chondrosarcomas of long bones and 48 years (range, 21-78 years) with a female-to-male ratio of $0.63: 1$ for pelvic chondrosarcomas. In long bones the most common location 
was the femur $(n=26)$ followed by the humerus $(n=11)$, tibia $(\mathrm{n}=2)$, and fibula $(\mathrm{n}=1)$.

Preoperative grading was assessed on a biopsy obtained with a Jamshidi needle under CT guidance. The number of samples obtained depended on the amount of tissue obtained with the first sample. If there was not a good tissue cylinder with the first sample, we repeated the procedure using the same approach. Postoperative grading was assessed after extensive sampling of surgical specimens. All histological samples were analyzed by two pathologists with experience in musculoskeletal pathology (PDR, AM) and in case of initial disagreement, a final consensus was established; for that reason in this study the inter- and intraobserver variability was not assessed.

All pelvic chondrosarcomas and high-grade chondrosarcomas of long bones were treated by en bloc resection. Low-grade chondrosarcomas of long bones were treated either by curettage or by en bloc resection after consensus from a multidisciplinary committee composed of pathologists, radiologists, and orthopaedic surgeons. The World Health Organization criteria were applied for histopathological diagnosis and grading [3]. Atypical cartilaginous tumor/Grade 1 chondrosarcomas are moderately cellular neoplasms and contain hyperchromatic plump nuclei of uniform size; Grade 2 are more cellular and contain a greater degree of nuclear atypia, hyperchromasia, and nuclear size; and Grade 3 lesions are more cellular and pleomorphic than Grade 2 with easily detected mitoses. Where the biopsy showed a mixed grade, the higher grade was taken as the final grading.

\section{Statistical Analysis}

The weighted and nonweighted kappa statistics were used to evaluate the agreement of tumor grading between the preoperative needle biopsy and the postoperative surgical specimen. The kappa coefficients for different localizations (pelvis and long bones) were compared using the Welch's t-test. The test was repeated in two different ways. First, the weighted kappa coefficient was computed for the three possible histological grades, both in the pelvis and long bones. Second, Grade 2 and Grade 3 chondrosarcomas were merged and the nonweighted kappa coefficient was calculated for pelvis and long bone data sets.

\section{Results}

Concordance between the preoperative biopsy and the final pathological analysis in terms of histological grade was much higher in long-bone chondrosarcoma than in pelvic chondrosarcoma (83\% [33 of 40] versus 36\% [14 of 39]; odds ratio [OR], 8,$48 ; \mathrm{p}<0.001)$. Likewise, the weighted kappa coefficients were higher in long-bone chondrosarcoma than in pelvic chondrosarcoma for the determination of histological grade $(0.63 ; 95 \%$ confidence interval [CI], $0.34-0.91$ versus $0.12 ;-0.32$ to $0.57 ; \mathrm{p}<0.001$ ).

In all discordant cases of long-bone chondrosarcoma (Table 1), a higher grade was found in the resection specimen (Fig. 1). In four of these patients, the grade moved from Grade 1 to Grade 2 and in three, a Grade 2 lesion was finally determined to be Grade 3 . In all cases but one of the 25 discordant pelvic chondrosarcomas, a higher grade was found in the resection specimen (Table 2).

When categorizing the lesions as low grade or high grade, concordance between the preoperative biopsy and the final pathological analysis was much higher in long-bone chondrosarcoma than in pelvic chondrosarcoma (90\% [36 of 40] versus $67 \%$ [26 of 39]; OR, 4, 5; $\mathrm{p}<0.001$ ). Likewise, the weighted kappa coefficients were higher in long-bone chondrosarcoma than in pelvic chondrosarcoma $(0.73 ; 95 \%$ CI, $0.51-0.94$ versus $0.26 ; 0.04-0.48 ; \mathrm{p}<0.001)$. In all discordant cases, the grade was underestimated in the biopsy.

\section{Discussion}

The diagnosis of cartilaginous lesions of the skeleton is one of the most challenging dilemmas in bone pathology.

Table 1. Summary of cases of chondrosarcoma of long bones with discrepancy in histological grade between preoperative needle biopsy and postoperative surgical specimen

\begin{tabular}{lllll}
\hline Gender & Age (years) & Preoperative biopsy & Surgical specimen & Affected bone \\
\hline Female & 57 & Atypical cartilaginous tumor/Grade 1 & Grade 2 & Femur \\
Male & 40 & Atypical cartilaginous tumor/Grade 1 & Grade 2 & Humerus \\
Male & 32 & Atypical cartilaginous tumor/Grade 1 & Grade 2 & Humerus \\
Female & 57 & Atypical cartilaginous tumor/Grade 1 & Grade 2 & Humerus \\
Female & 45 & Grade 2 & Grade 3 & Femur \\
Male & 53 & Grade 2 & Grade 3 & Femur \\
Male & 65 & Grade 2 & Grade 3 & Femur \\
\hline
\end{tabular}




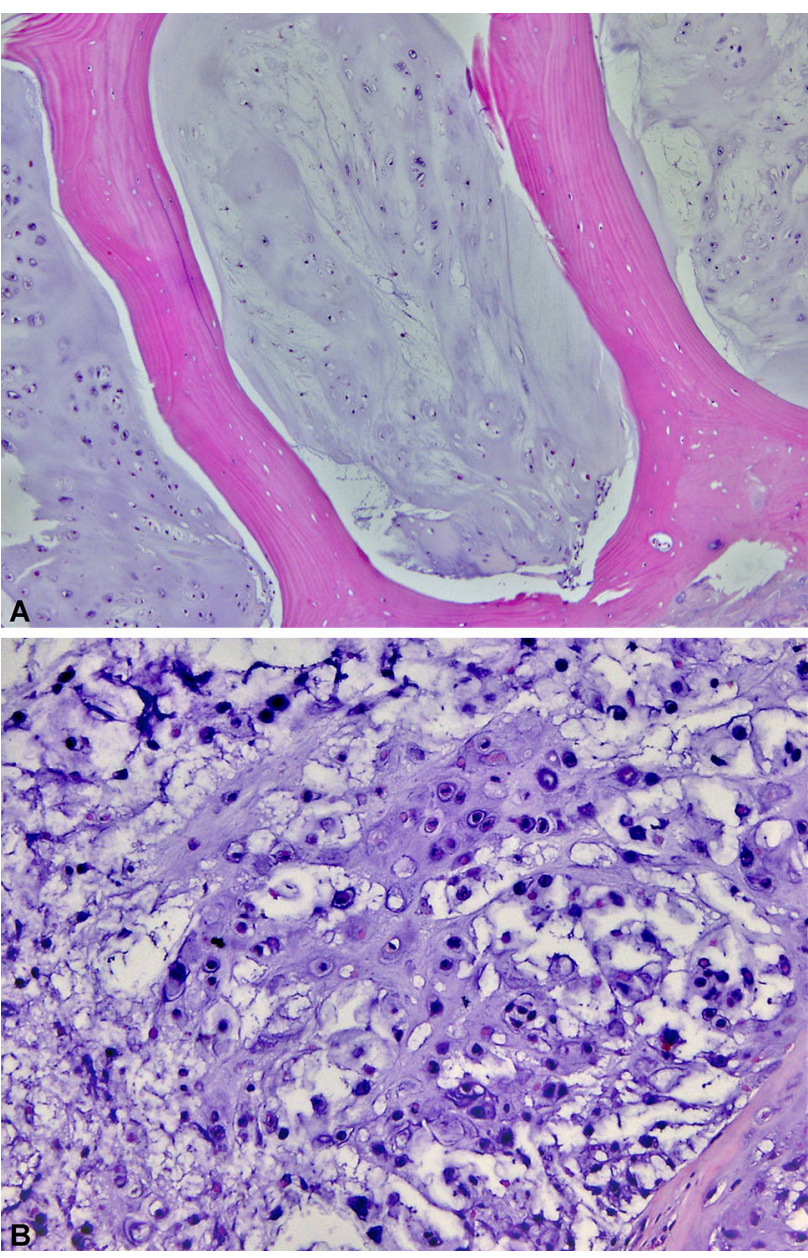

Fig. 1A-B Histological sections from the preoperative biopsy demonstrate a hypocellular but infiltrative cartilaginous neoplasm consistent with an atypical cartilaginous tumor/Grade 1 chondrosarcoma (Stain, hematoxylin-eosin; original magnification, $\times 100)(\mathbf{A})$. The resection specimen shows areas with myxoid matrix, increased cellularity, and a higher degree of nuclear pleomorphism, consistent with a Grade 2 chondrosarcoma (Stain, hematoxylin-eosin; original magnification, $\times 400)(\mathbf{B})$.

Although histological patterns for enchondroma and chondrosarcoma have been well described, they are not easy to recognize in small biopsies and the diagnosis is nearly always made with the support of clinical and radiological findings $[2,5,7,8,11,14,22,27]$. Not only can the distinction between benign and malignant be misleading, but also the grading of malignant lesions may differ significantly, even among experts [8,25]. Although some studies demonstrate that radiological imaging $[2,5,7]$ may be used to differentiate low-grade chondrosarcomas from highgrade ones, definitive grading still relies on histological features. This distinction is critical to avoid mistreatment. In this study, we found image-guided needle biopsy distinguished low from high grade in most cartilage tumors in long bones, but needle biopsy was not nearly as concordant for such tumors when they were located in the pelvis. Our study had some limitations. First, it was a retrospective study, and there are several disadvantages with this type of design: risk of error and/or biases in information related to the loss of cases, poor potential quality of collected information, and less statistical power. Second, we were not able to assess tumor size in each case, a variable that may be associated with the high number of discordant cases found in pelvic tumors. Finally, in this study the intraobserver and interoberver variability was not specifically assessed, although all samples were evaluated together by two pathologists with experience in musculoskeletal pathology. In case of initial disagreement, a final consensus was established after reviewing the case. We did not include radiological findings in this study. It is very clear that they play a crucial role in the differential diagnosis between enchondroma and low-grade chondrosarcoma and, as mentioned previously, may help in detecting high-grade chondrosarcomas. However, the purpose of this study was to look primarily at the preoperative biopsy in determining the tumor grade, which relies on histological criteria. It is beyond the scope of this work to discuss the differential diagnosis between enchondroma and chondrosarcoma or radiological parameters associated with high-grade chondrosarcomas. The main goal of this article is not to discuss our accuracy, as a group, in diagnosing chondrosarcomas (that would include clinical, radiological, and histological information), but to evaluate the weight of the information provided by needle biopsy (preoperative histological findings) in relation to establishing the grade of a chondrosarcoma.

The concordance between histological grade established on preoperative biopsy and the final pathological analysis was $83 \%$ for chondrosarcoma of long bones. Similar results are described in the literature, where concordance between histological grade in preoperative biopsy and the final pathological analysis varies from $65 \%$ to $86 \%[8,13,24$, $25,27]$. In our study, the concordance between needle biopsy and surgical specimen for histological grade, in pelvic chondrosarcomas, was poor (36\% [14 of 39]), in agreement with other published studies [15, 21]. In four chondrosarcomas of long bones initially diagnosed as low grade and treated by curettage, a second procedure was performed (en bloc resection) as a result of a final diagnosis of high grade (Grade 2). This inaccuracy points out the treacherous nature of cartilage tumors that in these four patients was misleading and led to potentially improper treatment, although we admit that we do not know what the course would have been if they had been observed closely for local recurrence. On the other hand, the preoperative biopsy correctly identified three high-grade tumors that were treated by resection; however, the final pathology on these three patients was reclassified from Grade 2 to Grade 3. This was an identified inaccuracy that did not affect our decision regarding treatment. 
Table 2. Summary of cases of chondrosarcoma of the pelvis with discrepancy in histological grade between preoperative needle biopsy and postoperative surgical specimen

\begin{tabular}{|c|c|c|c|}
\hline Gender & Age (years) & Preoperative biopsy & Surgical specimen \\
\hline Female & 21 & Atypical cartilaginous tumor/Grade 1 & Grade 2 \\
\hline Female & 36 & Atypical cartilaginous tumor/Grade 1 & Grade 2 \\
\hline Female & 29 & Atypical cartilaginous tumor/Grade 1 & Grade 2 \\
\hline Female & 65 & Atypical cartilaginous tumor/Grade 1 & Grade 2 \\
\hline Female & 65 & Atypical cartilaginous tumor/Grade 1 & Grade 2 \\
\hline Male & 72 & Atypical cartilaginous tumor/Grade 1 & Grade 2 \\
\hline Female & 31 & Atypical cartilaginous tumor/Grade 1 & Grade 2 \\
\hline Female & 37 & Atypical cartilaginous tumor/Grade 1 & Grade 3 \\
\hline Male & 68 & Atypical cartilaginous tumor/Grade 1 & Grade 3 \\
\hline Female & 55 & Atypical cartilaginous tumor/Grade 1 & Dedifferentiated \\
\hline Male & 53 & Atypical cartilaginous tumor/Grade 1 & Dedifferentiated \\
\hline Male & 51 & Atypical cartilaginous tumor/Grade 1 & Dedifferentiated \\
\hline Male & 53 & Atypical cartilaginous tumor/Grade 1 & Dedifferentiated \\
\hline Female & 40 & Grade 2 & Grade 3 \\
\hline Female & 43 & Grade 2 & Grade 3 \\
\hline Male & 67 & Grade 2 & Grade 3 \\
\hline Male & 68 & Grade 2 & Grade 3 \\
\hline Male & 69 & Grade 2 & Grade 3 \\
\hline Male & 37 & Grade 2 & Grade 3 \\
\hline Male & 37 & Grade 2 & Grade 3 \\
\hline Male & 68 & Grade 2 & Grade 3 \\
\hline Male & 29 & Grade 2 & Grade 3 \\
\hline Male & 58 & Grade 2 & Grade 3 \\
\hline Male & 38 & Grade 2 & Grade 3 \\
\hline Female & 56 & Grade 3 & Grade 2 \\
\hline
\end{tabular}

In $67 \%$ of pelvic chondrosarcomas, the preoperative biopsy and the final pathological analysis were concordant in terms of low grade or high grade. No second surgical procedures were performed in these patients because all had been treated with en bloc resection. Normand et al. [19] obtained similar results in eight patients with periacetabular chondrosarcomas initially diagnosed as low grade and treated with curettage. The final histology revealed that two of those tumors were best classified as Grade 2 and one was a dedifferentiated chondrosarcoma. Therefore, according to our findings, preoperative biopsy in the pelvis can have high-risk errors in tumor grade that may lead some surgeons to undertreatment (curettage) of a highgrade tumor with the risks that entails. However, it must be noted that most surgeons treat all cartilage tumors of the pelvis with complete resection and not curettage because of the perceived high rate of error and the adverse consequences of local recurrence.

The mismatches in grading can be partially explained considering the heterogeneity that chondrosarcomas demonstrate and the fact that only a limited amount of tissue can be obtained by needle biopsy, making it difficult to sample higher grade zones when they are not predominant in those tumors [21]. This diagnostic limitation becomes more important in large lesions and we think it may account for the much lower accuracy rate in pelvic chondrosarcomas, which are usually larger than their long bone counterparts [4, 20]. In this regard, a possible approach could be to use MRI to select specific areas of the tumor to be sampled. It has been shown that reduced signal intensity on T2-weighted MRI may represent foci of dedifferentiation and should be the preferred site of biopsy [23]. All of our biopsies were done by experienced musculoskeletal radiologists who had MRI findings in most cases and attempted to target the areas that looked by imaging to be the area of highest grade. Despite this, there was still substantial lack of concordance in pelvic cartilage tumors. There is little experience regarding intralesional treatment of low-grade chondrosarcomas of the pelvis and most surgeons prefer to resect rather than use curettage for pelvic cartilage tumors [19]. Donati et al. [6] reported that, although it led to an increased rate of local recurrences, it 
was not associated with poorer survival. It is important to emphasize that pelvic recurrences may end up infiltrating pelvic organs and structures, representing a more complex scenario than in the long bones. In any case, it should be stressed that all information including clinical findings, imaging findings, and biopsy results should be correlated by a sarcoma treatment team experienced in the treatment of these difficult lesions.

In conclusion, image-guided needle biopsy, when performed by a specialist radiologist and evaluated by an experienced bone pathologist, may be useful to the treating surgeon as a tool in determining the histological grade of long-bone chondrosarcomas allowing him or her to identify low-grade tumors from high-grade tumors in the long bones. In pelvic lesions, biopsy results should be interpreted with caution and may not be representative of the entire specimen. Larger studies will be necessary to confirm our results and treating surgeons should continue to make treatment decisions based on clinical, radiographic, and histologic findings in the long bones and be aware that a needle biopsy showing a low-grade chondrosarcoma of a pelvic lesion may be misleading. We have shown that in one-third of our pelvic patients, the needle biopsy results did not agree with the final pathology grading.

Acknowledgments We thank Dr Ana Morandi for her contribution in the histological diagnoses.

\section{References}

1. Andreou D, Ruppin S, Fehlberg S, Pink D, Werner M, Tunn PU. Survival and prognostic factors in chondrosarcoma: results in 115 patients with long-term follow-up. Acta Orthop. 2011;82:749755.

2. Berber O, Datta G, Sabharwal S, Aston W, Saifuddin A, Briggs T. The safety of direct primary excision of low-grade chondral lesions based on radiological diagnosis alone. Acta Orthop Belg. 2012;78:254-262.

3. Bertoni F, Bacchini P, Hogendoorn PCW. Chondrosarcoma. In: Fletcher CDM, Unni KK, Mertens F, eds. World Health Organization Classification of Tumours. Pathology and Genetics of Tumours of Soft Tissue and Bone. Lyon, France: IARC Press; 2002:247-251.

4. Bloem JL, Reidsma II. Bone and soft tissue tumors of hip and pelvis. Eur J Radiol. 2012;81:3793-3801.

5. Brown MT, Gikas PD, Bhamra JS, Skinner JA, Aston WJ, Pollock RC, Saifuddin A, Briggs TW. How safe is curettage of lowgrade cartilaginous neoplasms diagnosed by imaging with or without pre-operative needle biopsy? Bone Joint J. 2014;96:1098-1105.

6. Donati D, El Ghoneimy A, Bertoni F, Di Bella C, Mercuri M. Surgical treatment and outcome of conventional pelvic chondrosarcoma. J Bone Joint Surg Br. 2005;87:1527-1530.

7. Douis H, Singh L, Saifuddin A. MRI differentiation of low-grade from high-grade appendicular chondrosarcoma. Eur Radiol. 2014;24:232-240.
8. Eefting D, Schrage YM, Geirnaerdt MJ, Le Cessie S, Taminiau AH, Bovée JV, Hogendoorn PC; EuroBoNeT consortium. Assessment of interobserver variability and histologic parameters to improve reliability in classification and grading of central cartilaginous tumors. Am J Surg Pathol. 2009;33:50-57.

9. Enneking WF. A system of staging musculoskeletal neoplasms. Clin Orthop Relat Res. 1986;204:9-24.

10. Evans HL, Ayala AG, Romsdahl MM. Prognostic factors in chondrosarcoma of bone. A clinicopathologic analysis with emphasis on histologic grading. Cancer. 1977;40:818-831.

11. Ferrer-Santacreu EM, Ortiz-Cruz EJ, González-López JM, Pérez Fernández E. Enchondroma versus low-grade chondrosarcoma in appendicular skeleton: clinical and radiological criteria. J Oncol. 2012;2012:437958.

12. Funovics PT, Panotopoulos J, Sabeti-Aschraf M, Abdolvahab F, Funovics JM, Lang S, Kotz RI, Dominkus M. Low-grade chondrosarcoma of bone: experiences from the Vienna Bone and Soft Tissue Tumour Registry. Int Orthop. 2011;35:1049-1056.

13. Jennings R, Riley N, Rose B, Rossi R, Skinner JA, Cannon SR, Briggs TW, Pollock R, Saifuddin A. An evaluation of the diagnostic accuracy of the grade of preoperative biopsy compared to surgical excision in chondrosarcoma of the long bones. Int J Surg Oncol. 2010;2010:270195.

14. Kendell SD, Collins MS, Adkins MC, Sundaram M, Unni KK. Radiographic differentiation of enchondroma from low-grade chondrosarcoma in the fibula. Skeletal Radiol. 2004;33:458-466.

15. Konishi E, Nakashima Y, Mano M, Tomita Y, Nagasaki I, Kubo T, Araki N, Haga H, Toguchida J, Ueda T, Sakuma T, Imahori M, Morii E, Yoshikawa H, Tsukamoto Y, Futani H, Wakasa K, Hoshi M, Hamada S, Takeshita H, Inoue T, Aono M, Kawabata K, Murata H, Katsura K, Urata Y, Ueda H, Yanagisawa A. Primary central chondrosarcoma of long bone, limb girdle and trunk: analysis of 174 cases by numerical scoring on histology. Pathol Int. 2015;65:468-475.

16. Lee FY, Mankin HJ, Fondren G, Gebhardt MC, Springfield DS, Rosenberg AE, Jennings LC. Chondrosarcoma of bone: an assessment of outcome. J Bone Joint Surg Am. 1999;81:326-338.

17. Mavrogenis AF, Angelini A, Drago G, Merlino B, Ruggieri P. Survival analysis of patients with chondrosarcomas of the pelvis. J Surg Oncol. 2013;108:19-27.

18. Meftah M, Schult P, Henshaw RM. Long-term results of intralesional curettage and cryosurgery for treatment of low-grade chondrosarcoma. J Bone Joint Surg Am. 2013;95:1358-1364.

19. Normand AN, Cannon CP, Lewis VO, Lin PP, Yasko AW. Curettage of biopsy-diagnosed grade 1 periacetabular chondrosarcoma. Clin Orthop Relat Res. 2007;459:146-149.

20. Pring ME, Weber KL, Unni KK, Sim FH. Chondrosarcoma of the pelvis: a review of sixty-four cases. J Bone Joint Surg Am. 2001;11:1630-1642.

21. Rinas AC, Ward WG, Kilpatrick SE. Potential sampling error in fine needle aspiration biopsy of dedifferentiated chondrosarcoma: a report of 4 cases. Acta Cytol. 2005;49:554-559.

22. Rosenthal DI, Schiller AL, Mankin HJ. Chondrosarcoma: correlation of radiological and histological grade. Radiology. 1984;150:21-26.

23. Saifuddin A, Mann BS, Mahroof S, Pringle JA, Briggs TW, Cannon SR. Dedifferentiated chondrosarcoma: use of MRI to guide needle biopsy. Clin Radiol. 2004;59:268-272.

24. Sanerkin NG. The diagnosis and grading of chondrosarcoma of bone: a combined cytologic and histologic approach. Cancer. 1980;45:582-594.

25. Skeletal Lesions Interobserver Correlation among Expert Diagnosticians (SLICED) Study Group. Reliability of histopathologic 
and radiologic grading of cartilaginous neoplasms in long bones. J Bone Joint Surg Am. 2007;89:2113-2123.

26. Verdegaal SH, Brouwers HF, van Zwet EW, Hogendoorn PC, Taminiau AH. Low-grade chondrosarcoma of long bones treated with intralesional curettage followed by application of phenol, ethanol, and bone-grafting. J Bone Joint Surg Am. 2012;94:12011207.

27. Yoshimura Y, Isobe K, Arai H, Aoki K, Kito M, Kato H. Preoperative radiographic and histopathologic evaluation of central chondrosarcoma. Arch Orthop Trauma Surg. 2013;133:1225-1231. 